\title{
Energy Detection under RF impairments for Cognitive Radio
}

\author{
Alexandros-Apostolos A. Boulogeorgos*, Nestor D. Chatzidiamantis*, George K. Karagiannidis* \\ and Leonidas Georgiadis* \\ * Department of Electrical and Computer Engineering, Aristotle University of Thessaloniki, Greece, Emails:\{ampoulog,nestoras,geokarag,leonid\}@auth.gr
}

\begin{abstract}
Direct-conversion radio receivers can offer highly integrated low-cost hardware solutions for spectrum sensing in cognitive radio systems. However, these receivers are susceptible to radio frequency (RF) impairments, such as in-phase and quadrature-phase imbalance, low-noise amplifier nonlinearities and phase noise, which limit the spectrum sensing capabilities. In this paper, we study the joint effects of RF impairments on energy detection based spectrum sensing in multi-channel environments. In particular, we provide an analytical framework for the evaluation of the probabilities of detection and false alarm, assuming Rayleigh fading and approximating the joint effects of RF impairments by a Gaussian distribution. Numerical results illustrate the detrimental effects of $R F$ imperfections on the spectrum sensing performance, which bring significant losses in the spectrum utilization.
\end{abstract}

\section{INTRODUCTION}

The rapid growth of wireless communications and the foreseen spectrum occupancy problems, due to the exponentially increasing consumer demands on mobile traffic and data, motivated the evolution of the concept of cognitive radio (CR) [1]. One important task in CR is spectrum sensing, i.e., the identification of temporarily vacant portions of spectrum, which allows the exploitation of the under-utilized spectrum and is considered to be an essential element in the operation of CRs. Therefore, great amount of effort has been put to derive optimal, suboptimal and ad-hoc solutions to the spectrum sensing problem (see for example [2]-[7] and the references therein). However, the majority of these works ignore the imperfections associated with the RF front-end (FE). Such imperfections, which are encountered in the widely deployed direct-detection radio receivers, include in-phase and quadrature-phase (I/Q) imbalance (IQI) [8], low-noise amplifier (LNA) nonlinearities [9] and phase noise (PHN) [10].

Only recently, the impacts of RF impairments in the spectrum sensing capabilities of CR have been investigated [8], [10]-[14]. In particular, the impacts of RF impairments in direct-conversion receivers on single-channel energy and/or cyclostationary based sensing was discussed in [11], [12]. Furthermore, in [13] the authors derived closed-form expressions for the detection and false alarm probabilities for NeymanPearson detector, considering the spectrum sensing problem in single-channel orthogonal frequency division multiplexing (OFDM) CR receiver, under joint transmitter and receiver IQI. Multi-channel sensing under IQI was reported in [14], where a three-level hypothesis blind detector was introduced. Moreover, the impact of RF IQI on energy detection (ED) for both single-channel and multi-channel direct-conversion receivers was investigated in [8], where it was shown that the false alarm probability in a multi-channel environment increases significantly, compared to the ideal RF receiver case. Furthermore, in [10], the authors analyzed the effect of PHN on ED, considering a multi-channel direct-conversion receiver and AWGN channels.

In this work, we investigate the impact on the multi-channel energy-based spectrum sensing mechanism of the joint effects of several RF impairments, such as LNA non-linearities, PHN and IQI. After assuming flat-fading Rayleigh channels and complex Gaussian transmitted signals, and approximating the joint effects of RF impairments by a complex Gaussian process (an approximation which has been validated both in theory and by experiments, see [15] and the references therein), we derive analytical expressions for the probabilities of false alarm and detection. Based on these expressions, we investigate the impact of RF impairments on ED. Specifically, the contribution of this paper can be summarized as follows:

- We, first, derive analytical expressions for the false alarm and detection probabilities for an ideal ED detector, assuming flat fading Rayleigh channels and complex Gaussian transmitted signals. To the best of the authors' knowledge, this is the first time that such expressions are presented in the open literature, under these assumptions.

- A signal model that describes the joint effects of all RF impairments is presented. This model is built upon an approximation of the joint effects of RF impairments by a complex Gaussian process [15] and is tractable to algebraic manipulations.

- An analytical framework is provided for the evaluation of false alarm and detection probabilities of multi-channel EDs constrained by RF impairments, under Rayleigh fading. Based on this framework, the joint effects of $\mathrm{RF}$ impairments on spectrum sensing performance is investigated.

Notations: Unless otherwise stated, $\Re\{x\}$ and $\Im\{x\}$ represent the real and imaginary part of $x$, respectively. The operators $E[\cdot]$ and $|\cdot|$ denote the statistical expectation and the absolute value, respectively. The sign of a real number $x$ is returned by the operator $\operatorname{sign}(x)$. The operator card $(\mathcal{A})$ returns the cardinality of the set $\mathcal{A}$. The lower [16, Eq. $(8.350 / 1)]$ and upper incomplete Gamma functions [16, Eq. $(8.350 / 2)]$ are represented by $\gamma(\cdot, \cdot)$ and $\Gamma(\cdot, \cdot)$, respectively, 
while the Gamma function [16, Eq. 8.310)] is denoted by $\Gamma(\cdot) . \mathrm{Q}(x)=\frac{1}{\sqrt{2 \pi}} \int_{x}^{\infty} \exp \left(-t^{2} / 2\right) d t$ is the Gaussian Qfunction. $K_{\nu}(\cdot)$ is the $\nu$-th order modified Bessel function of the second kind [16, Eq. (8.432/9)] and $G_{p, q}^{m, n}[\cdot]$ is the Meijer's $G$-function [16, Eq.9.301/1].

\section{SYSTEM AND SIGNAL MODEL}

\section{A. Ideal RF front-end}

Next, we present the signal model for the multi-channel sensing scenario under ideal RF FE setting. The two hypothesis, namely absence/presence of primary user (PU), is denoted with parameter $\theta_{k} \in\{0,1\}$.

Suppose a PU signal, $s(n)$, is conveyed over a flatfading wireless channel, $h(n)$, with additive noise $w(n)$. The received wideband RF signal is passed through various RF FE stages, including filtering, amplification, analog I/Q demodulation (down-conversion) to baseband and sampling. The wideband channel after sampling is assumed to have a bandwidth of $W$ and contain $K$ channels, each having bandwidth $W_{c h}=W_{s b}+W_{g b}$, where $W_{s b}$ and $W_{g b}$ are the signal band and total guard band bandwidth within this channel, respectively. Additionally, it is assumed that the sampling is performed with rate $W$.

After the selection filter, the baseband equivalent received signal vector for the $k^{\text {th }}$ channel $(k \in$ $S\{-K / 2, \ldots,-1,1 \ldots, K / 2\})$ is given by

$$
\begin{aligned}
r_{k}(n) & =\Re\left\{r_{k}(n)\right\}+j \Im\left\{r_{k}(n)\right\} \\
& =\theta_{k} h_{k}(n) s_{k}(n)+w_{k}(n),
\end{aligned}
$$

where $h_{k}, s_{k}$ and $w_{k}$ are zero-mean CSCWG processes with variances $\sigma_{h}^{2}, \sigma_{s}^{2}$ and $\sigma_{w}^{2}$, respectively.

Note, that the rate of the signal is reduced by a factor of $L=W / W_{s b} \geq K$, where for simplicity we assume $L \in \mathbb{Z}$.

\section{B. Non-ideal RF front-end}

The direct-conversion wideband sensing scenario is very sensitive to RF circuit impairments, such as LNA nonlinearities, PHN and IQI.

The impaired baseband equivalent received signal vector for the $k^{\text {th }}$ channel is given by [8], [17]

$$
\begin{aligned}
r_{k}(n) & =\Re\left\{r_{k}(n)\right\}+j \Im\left\{r_{k}(n)\right\} \\
& =\xi_{k}(n) \theta_{k} h_{k}(n) s_{k}(n)+\eta_{k}(n)+w_{k}(n),
\end{aligned}
$$

where $\xi_{k}$ stands for the amplitude and phase rotation due to PHN caused by common phase error (CPE), LNA nonlinearities and IQI, and is given by

$$
\xi_{k}=\gamma_{0} K_{1} \alpha,
$$

while $\eta_{k}$ denotes the distortion noise from impairments in the receiver, due to PHN caused by inter carrier interference (ICI), IQI and non-linear distortion noise, and is given by

$$
\begin{aligned}
& \eta_{k}(n)=K_{1}\left(\gamma_{o} e_{k}(n)+\psi_{k}(n)\right) \\
& +K_{2}\left(\gamma_{o}^{*}\left(\alpha h_{-k}^{*}(n) s_{-k}^{*}(n)+e_{-k}^{*}(n)\right)+\psi_{-k}^{*}(n)\right) .
\end{aligned}
$$

After denoting as $\Theta_{k}=\left\{\theta_{k-1}, \theta_{k+1}\right\}$ and $H_{k}=$ $\left\{h_{k-1}, h_{k+1}\right\}$, this distortion noise term can be modeled as $\eta_{k} \sim \mathcal{C N}\left(0, \sigma_{\eta_{k}}^{2}\right)$, with

$$
\begin{aligned}
\sigma_{\eta_{k}}^{2} & =\left|\gamma_{0}\right|^{2}\left(\left|K_{1}\right|^{2} \sigma_{e, k}^{2}+\left|K_{2}\right|^{2} \sigma_{e,-k}^{2}\right) \\
& +\left|K_{1}\right|^{2} \sigma_{\psi \mid H_{k}, \Theta_{k}}^{2}+\left|K_{2}\right|^{2} \sigma_{\psi \mid H_{-k}, \Theta_{-k}}^{2} \\
& +\left|\gamma_{0}\right|^{2}\left|K_{2}\right|^{2}|\alpha|^{2} \theta_{-k}\left|h_{-k}\right|^{2} \sigma_{s}^{2} .
\end{aligned}
$$

Note that this model has been supported and validated by many theoretical investigations and measurements [15]. Next, we describe how the various parameters in (5), (6) and (7) stem from the imperfections associated with the non-ideal RF FE.

LNA Nonlinearities: The parameters $\alpha$ and $e_{k}$ respresent the nonlinearity parameters, which model the amplitude/phase distortion and the nonlinear distortion noise, respectively. According to Bussgang's theorem [18], $e_{k}$ is a zero-mean Gaussian error term with variance $\sigma_{e_{k}}^{2}$. Considering an ideal clipping power amplifier (PA), the amplification factor $\alpha$ and the variance $\sigma_{e_{k}}^{2}$, are given by

$$
\begin{gathered}
\alpha=1-\exp (-\mathrm{IBO})+\sqrt{2 \pi} \mathrm{IBO} \mathrm{Q}(2 \mathrm{IBO}), \\
\sigma_{e_{k}}^{2}=\sigma_{s}^{2}\left(1-\alpha^{2}-\exp (-\mathrm{IBO})\right),
\end{gathered}
$$

where IBO $=A_{o}^{2} / \sigma_{s}^{2}$ denotes the input back-off factor and $A_{o}$ is the PA's clipping level.

I/Q Imbalance: The IQI coefficients $K_{1}$ and $K_{2}$ are given by

$$
K_{1}=\frac{1+\epsilon e^{-j \theta}}{2} \text { and } K_{2}=\frac{1-\epsilon e^{j \theta}}{2},
$$

with $\epsilon$ and $\theta$ denote the amplitude and phase mismatch, respectively. It is noted that for perfect $\mathrm{I} / \mathrm{Q}$ matching, this imbalance parameters become $\epsilon=1, \theta=0$; thus in this case $K_{1}=1$ and $K_{2}=0$. The coefficients $K_{1}$ and $K_{2}$ are related through the image rejection ratio (IRR) is given by IRR $=\left|K_{1} / K_{2}\right|^{2}$. With practical analog FE electronics, IRR is typically in the range of $25-40 \mathrm{~dB}$ [19], [20].

Phase noise: The parameter $\gamma_{0}$ stands for CPE, which is equal for all carriers, and $\psi_{k}$ represents the ICI from all other neighboring channels due to spectral regrowth caused by PHN. However, the typical $3 \mathrm{~dB}$ bandwidth values for the oscillator process is in the order of few tens or hundreds of $\mathrm{Hz}$, with rapidly fading spectrum after this point (approximately $10 \mathrm{~dB} /$ decade). Thus, for channel bandwidth that is typical few tens or hundreds $\mathrm{KHz}$, then the only effective interference is due to leakage from successive neighbors only. Hence, the ICI term can be approximated as [10]

$$
\begin{aligned}
\psi_{k}(n) & \approx \theta_{k-1} \gamma(n) h_{k-1}(n) s_{k-1}(n) \\
& +\theta_{k+1} \gamma(n) h_{k+1}(n) s_{k+1}(n),
\end{aligned}
$$

with $\gamma(n)=e^{j \phi(n)}$ and $\phi(n)$ being a discrete Brownian error process, i.e., $\phi(n)=\sum_{m=1}^{n} \phi(m-1)+\epsilon(n)$, where $\epsilon(n)$ is a zero mean real Gaussian variable with variance $\sigma_{\epsilon}^{2}=$ $4 \pi \beta / W$ and $\beta$ being the $3-\mathrm{dB}$ bandwidth of the oscillator process. 
The interference term $\psi_{k}$ in (6) might have zero or nonzero contribution depending on the existence of PU signals in the successive neighboring channels. In general, this term is typically non-white and strictly speaking cannot be modeled by a Gaussian process. However, for practical $3 d B$ bandwidth of the oscillator process, the influence of the regarded impairments can all be modeled as a zero-mean Gaussian process with $\sigma_{\psi_{k} \mid\left\{H_{k}, \Theta_{k}\right\}}^{2}$ given by

$$
\begin{aligned}
\sigma_{\psi \mid\left\{H_{k}, \Theta_{k}\right\}}^{2} & =\theta_{k-1} A_{k-1}\left|h_{k-1}(n)\right|^{2} \sigma_{s}^{2} \\
& +\theta_{k+1} A_{k+1}\left|h_{k+1}(n)\right|^{2} \sigma_{s}^{2},
\end{aligned}
$$

where

$$
\begin{aligned}
& A_{k-1}=\frac{\left|I\left(f_{k-1}-f_{k}+f_{\text {cut-off }}\right)-I\left(f_{k-1}-f_{k}-f_{\text {cut-off }}\right)\right|}{2 \pi f_{\text {cut-off }}}, \\
& A_{k+1}=\frac{\left|I\left(f_{k+1}-f_{k}+f_{\text {cut-off }}\right)-I\left(f_{k+1}-f_{k}-f_{\text {cut-off }}\right)\right|}{2 \pi f_{\text {cut-off }}},
\end{aligned}
$$

and $f_{k}$ is the centered normalized frequency of the $k^{\text {th }}$ channel, i.e., $f_{k}=\operatorname{sign}(k) \frac{2|k|-1}{2 K}$ and $f_{\text {cut-off }}=\frac{W_{s b}}{2 W}$. Furthermore,

$$
I(f)=B(f)-C(f),
$$

where

$$
\begin{aligned}
B(f) & =\left(f_{\text {cut-off }}-f\right) \tan ^{-1}\left(\delta \tan \left(\pi\left(f_{\text {cut-off }}-f\right)\right)\right) \\
& +\left(f_{\text {cut-off }}+f\right) \tan ^{-1}\left(\delta \tan \left(-\pi\left(f_{\text {cut-off }}+f\right)\right)\right), \\
C(f) & =\frac{1}{\delta}\left(\left(f_{\text {cut-off }}+f\right) \cot \left(\pi\left(f_{\text {cut-off }}+f\right)\right)\right. \\
& \left.-\left(f_{\text {cut-off }}-f\right) \cot \left(\pi\left(f_{\text {cut-off }}-f\right)\right)\right) \\
& -\frac{1}{\pi \delta}\left(\log \left(\left|\sin \left(\pi\left(f_{\text {cut-off }}+f\right)\right)\right|\right)\right. \\
& \left.+\log \left(\left|\sin \left(\pi\left(f_{\text {cut-off }}-f\right)\right)\right|\right)\right)
\end{aligned}
$$

and $\delta=\left(e^{-2 \pi \beta / W}+1\right) /\left(e^{-2 \pi \beta / W}-1\right)$. Note that due to Eqs. (13) and (14), it follows that $A_{k-1}=A_{k+1}$.

\section{False Alarm/Detection Probabilities for CHANNEL DETECTION}

In the classical ED, the energy of the received signals is used to determine if a channel is empty or busy. Based on the signal model described above, the ED calculates the test statistics for the $k$ th channel as

$$
\begin{aligned}
T_{k} & =\frac{1}{N_{s}} \sum_{m=0}^{N_{s}-1}\left|r_{k}(n-m)\right|^{2} \\
& =\frac{1}{N_{s}} \sum_{m=0}^{N_{s}-1} \Re\left\{r_{k}(n-m)\right\}^{2}+\Im\left\{r_{k}(n-m)\right\}^{2},
\end{aligned}
$$

where $N_{s}$ is the number of complex samples used for sensing. This test statistic is compared against a threshold $\gamma_{t h}$ to yield the sensing decision, i.e. $k$-th channel busy if $T_{k}>\gamma_{t h}$ or idle otherwise.

\section{A. Ideal RF front-end}

Based on the signal model presented in II-A and taking into consideration that

$$
\begin{aligned}
\sigma^{2} & =E\left[\Re\left\{r_{k}\right\}^{2}\right]=E\left[\Im\left\{r_{k}\right\}^{2}\right] \\
& =\theta_{k}\left(\Re\left\{h_{k}\right\}^{2}+\Im\left\{h_{k}\right\}^{2}\right) \frac{\sigma_{s}^{2}}{2}+\frac{\sigma_{w}^{2}}{2},
\end{aligned}
$$

and $E\left[\Re\left\{r_{k}\right\} \Im\left\{r_{k}\right\}\right]=0$ for a given channel realization $h_{k}$ and channel occupation $\theta_{k}$, the received energy follows chi-square distribution with $2 N_{s}$ degrees of freedom and cumulative distribution function (CDF) given by

$$
F_{T_{k}}\left(x \mid h_{k}, \theta_{k}\right)=\frac{\gamma\left(N_{s}, \frac{N_{s} x}{2 \sigma^{2}}\right)}{\Gamma\left(N_{s}\right)} .
$$

The following theorem returns an analytical expression for the $\mathrm{CDF}$ of the test statistics assuming that the channel is busy.

Theorem 1. The CDF of the energy statistics assuming an ideal RF front end and busy channel can be evaluated by

$$
\begin{aligned}
& F_{T_{k}}\left(x \mid \theta_{k}=1\right)=\exp \left(\frac{\sigma_{w}^{2}}{\sigma_{2}^{2} \sigma_{h}^{2}}\right) \\
& -\frac{2 \exp \left(\frac{\sigma_{w}^{2}}{\sigma_{s}^{2} \sigma_{h}^{2}}\right)}{\Gamma\left(N_{s}\right)}\left(\frac{N_{s} x}{\sigma_{h}^{2} \sigma_{s}^{2}}\right)^{\frac{N_{s}}{2}} K_{N_{s}}\left(2 \sqrt{\frac{N_{s} x}{\sigma_{h}^{2} \sigma_{s}^{2}}}\right) \\
& -\exp \left(\frac{\sigma_{w}^{2}}{\sigma_{s}^{2} \sigma_{h}^{2}}\right)+1+\frac{\exp \left(\frac{\sigma_{w}^{2}}{\sigma_{s}^{2} \sigma_{h}^{2}}\right)}{\sigma_{s}^{2} \sigma_{h}^{2}} \\
& \times \sum_{j=0}^{N_{s}-1} \frac{\left(N_{s} x\right)^{j}}{j ! 2^{j-1}} \int_{0}^{\frac{\sigma_{w}^{2}}{2}} y^{-j} \exp \left(\frac{N_{s} x}{2 y}\right) \exp \left(-\frac{2 y}{\sigma_{h}^{2} \sigma_{s}^{2}}\right) d y .
\end{aligned}
$$

Proof: Since $h_{k} \sim \mathcal{C N}\left(0, \sigma_{h}^{2}\right)$, it follows that the parameter $\sigma^{2}$ follows exponential distribution with probability density function (PDF) given by

$$
f_{\sigma^{2}}\left(x \mid \theta_{k}=1\right)=\frac{2 \exp \left(\frac{\sigma_{w}^{2}}{\sigma_{s}^{2} \sigma_{h}^{2}}\right)}{\sigma_{s}^{2} \sigma_{h}^{2}} \exp \left(-\frac{2 x}{\sigma_{s}^{2} \sigma_{h}^{2}}\right),
$$

with $x \in\left[\frac{\sigma_{w}^{2}}{2}, \infty\right)$. Hence, the unconditional CDF can be written as

$$
\begin{aligned}
F_{T_{k}}\left(x \mid \theta_{k}=\right. & 1)=\frac{1}{\Gamma\left(N_{s}\right)} \frac{2 \exp \left(\frac{\sigma_{w}^{2}}{\sigma_{s}^{2} \sigma_{h}^{2}}\right)}{\sigma_{s}^{2} \sigma_{h}^{2}} \\
& \times \int_{\frac{\sigma_{w}^{2}}{2}}^{\infty} \gamma\left(N_{s}, \frac{N_{s} x}{2 y}\right) \exp \left(-\frac{2 y}{\sigma_{h}^{2} \sigma_{s}^{2}}\right) d y
\end{aligned}
$$

which is equivalent to

$$
\begin{aligned}
& F_{T_{k}}\left(x \mid \theta_{k}=1\right)=\frac{2 \exp \left(\frac{\sigma_{w}^{2}}{\sigma_{s}^{2} \sigma_{h}^{2}}\right)}{\sigma_{s}^{2} \sigma_{h}^{2}} \int_{0}^{\infty} \exp \left(-\frac{2 y}{\sigma_{h}^{2} \sigma_{s}^{2}}\right) d y \\
& -\frac{2 \exp \left(\frac{\sigma_{w}^{2}}{\sigma_{s}^{2} \sigma_{h}^{2}}\right)}{\sigma_{s}^{2} \sigma_{h}^{2} \Gamma\left(N_{s}\right)} \int_{0}^{\infty} \Gamma\left(N_{s}, \frac{N_{s} x}{2 y}\right) \exp \left(-\frac{2 y}{\sigma_{h}^{2} \sigma_{s}^{2}}\right) d y \\
& -\frac{2 \exp \left(\frac{\sigma_{w}^{2}}{\sigma_{s}^{2} \sigma_{h}^{2}}\right)}{\sigma_{s}^{2} \sigma_{h}^{2} \Gamma\left(N_{s}\right)} \int_{0}^{\frac{\sigma_{w}^{2}}{2}} \gamma\left(N_{s}, \frac{N_{s} x}{2 y}\right) \exp \left(-\frac{2 y}{\sigma_{h}^{2} \sigma_{s}^{2}}\right) d y .
\end{aligned}
$$


After some algebraic manipulations, using [16, Eq. (6.453)] and [16, Eq. (8.352/1)], (25) can be written as in (22). This concludes the proof.

It should be noted that the calculation of an integral, composed of elementary functions and with finite limits, is involved in the evaluation of (22). This operation can be efficiently performed with numerical integration methods.

Based on the above analysis, the false alarm probability for the ideal receiver can be calculated by

$$
\mathcal{P}_{F A}=1-\frac{\gamma\left(N_{s}, \frac{N_{s} \gamma_{t h}}{\sigma_{w}^{2}}\right)}{\Gamma\left(N_{s}\right)},
$$

while the probability of detection is given by

$$
\mathcal{P}_{D}=1-F_{T_{k}}\left(\gamma_{t h} \mid \theta_{k}=1\right) .
$$

\section{B. Non-Ideal RF Front-End}

Based on the signal model presented in II-B and assuming given channel realization and channel occupancy vectors $H=\left\{H_{-k}, h_{-k}, h_{k}, H_{k}\right\}$ and $\Theta=\left\{\Theta_{-k}, \theta_{-k}, \theta_{k}, \Theta_{k}\right\}$, respectively, it holds that

$$
\begin{aligned}
\sigma^{2} & =E\left[\Re\left\{r_{k}\right\}^{2}\right]=E\left[\Im\left\{r_{k}\right\}^{2}\right] \\
& =\theta_{k}\left(\Re\left\{h_{k}\right\}^{2}+\Im\left\{h_{k}\right\}^{2}\right)\left(\Re\left\{\xi_{k}\right\}^{2}+\Im\left\{\xi_{k}\right\}^{2}\right) \frac{\sigma_{s}^{2}}{2} \\
& +\frac{\sigma_{w}^{2}+\sigma_{\eta_{k}}^{2}}{2}
\end{aligned}
$$

and $E\left[\Re\left\{r_{k}\right\} \Im\left\{r_{k}\right\}\right]=0$. Thus, the received energy, given by (19), follows chi-square distribution with $2 N_{s}$ degrees of freedom and CDF given by

$$
F_{T_{k}}(x \mid H, \Theta)=\frac{\gamma\left(N_{s}, \frac{N_{s} x}{2 \sigma^{2}}\right)}{\Gamma\left(N_{s}\right)},
$$

where $\sigma^{2}$ can be expressed, after taking into account (28),(7) and (12), as

$$
\begin{aligned}
& \sigma^{2}=\theta_{k} \mathcal{A}_{1}\left|h_{k}\right|^{2}+\theta_{k-1} \mathcal{A}_{2}\left|h_{k-1}\right|^{2}+\theta_{k+1} \mathcal{A}_{2}\left|h_{k+1}\right|^{2} \\
& +\theta_{-k+1} \mathcal{A}_{3}\left|h_{-k+1}\right|^{2}+\theta_{-k+1} \mathcal{A}_{3}\left|h_{-k-1}\right|^{2} \\
& +\theta_{-k} \mathcal{A}_{4}\left|h_{-k}\right|^{2}+\mathcal{A}_{5}
\end{aligned}
$$

with $\mathcal{A}_{1}=\left|\xi_{k}\right|^{2} \frac{\sigma_{s}^{2}}{2}, \mathcal{A}_{2}=\left|K_{1}\right|^{2} A_{k-1} \frac{\sigma_{s}^{2}}{2}, \mathcal{A}_{3}=$ $\left|K_{2}\right|^{2} A_{-k+1} \frac{\sigma_{s}^{2}}{2}, \mathcal{A}_{4}=\left|\gamma_{0}\right|^{2}\left|K_{2}\right|^{2}|a|^{2} \frac{\sigma_{s}^{2}}{2}$, and $\mathcal{A}_{5}=\frac{\sigma_{w}^{2}}{2}+$ $\frac{\left|\gamma_{0}\right|^{2}}{2}\left(\left|K_{1}\right|^{2} \sigma_{e, k}^{2}+\left|K_{2}\right|^{2} \sigma_{e,-k}^{2}\right)$.

Theorem 2. The CDF of the energy statistics assuming an non-ideal RF front end and if the channel occupancy vector $\Theta$ is different than the all idle vector can be evaluated as in (34) at the top of the next page, where

$$
\begin{gathered}
m=\left[\theta_{k}, \theta_{k-1}+\theta_{k+1}, \theta_{-k+1}+\theta_{-k-1}, \theta_{-k}\right], \\
w_{1, i}=\frac{\exp \left(\frac{\mathcal{A}_{5}}{\mathcal{A}_{i}}\right)}{\Gamma\left(m_{i}\right)\left(\prod_{j=1}^{4} \mathcal{A}_{j}^{m_{j}}\right)} \prod_{j=1, j \neq i}^{4}\left(\frac{1}{\mathcal{A}_{j}}-\frac{1}{\mathcal{A}_{i}}\right)^{-m_{j}}, \\
\text { and } w_{2, i}=\sum_{j=1, j \neq i} m_{j}\left(\frac{1}{\mathcal{A}_{j}}-\frac{1}{\mathcal{A}_{i}}\right)^{-1} .
\end{gathered}
$$

Proof: According to [21] and after some basic algebraic manipulations, its PDF can be written as

$$
\begin{aligned}
& f_{\sigma^{2}}(x \mid \Theta)=\sum_{i=2}^{3} U\left(m_{i}-2\right) w_{1, i} w_{2, i} \exp \left(-\frac{x}{\mathcal{A}_{i}}\right) \\
& +\sum_{i=1}^{4} U\left(m_{i}-1\right) U\left(m_{i}-2\right) w_{1, i} x \exp \left(-\frac{x}{\mathcal{A}_{i}}\right) \\
& +\sum_{i=1}^{4} U\left(m_{i}-1\right)\left(U\left(1-m_{i}\right)-\mathcal{A}_{5} U\left(m_{i}-2\right)\right) \\
& \times w_{1, i} \exp \left(-\frac{x}{\mathcal{A}_{i}}\right),
\end{aligned}
$$

where $m, w_{1, i}$ and $w_{2, i}$ are defined by (31), (32) and (33) respectively, $U(x)$ is the well-known unit step function, with $x \in\left[\mathcal{A}_{5}, \infty\right)$.

Based on the above, the CDF of the received energy, in case of non-ideal RF FE, unconditioned with respect to $\Theta$, is given by

$$
\begin{aligned}
& F_{T_{k}}(x \mid \Theta)=\sum_{i=2}^{3} U\left(m_{i}-2\right) w_{1, i} w_{2, i} \mathcal{I}_{1, i}, \\
& \quad+\sum_{i=1}^{4} U\left(m_{i}-1\right)\left(U\left(1-m_{i}\right)-\mathcal{A}_{5} U\left(m_{i}-2\right)\right) w_{1, i} \mathcal{I}_{1, i} \\
& \quad+\sum_{i=1}^{4} U\left(m_{i}-1\right) U\left(m_{i}-2\right) w_{1, i} \mathcal{I}_{2, i}
\end{aligned}
$$

with

$$
\begin{aligned}
& \mathcal{I}_{1, i}=\frac{1}{\Gamma\left(N_{s}\right)} \int_{\mathcal{A}_{5}}^{\infty} \exp \left(-\frac{y}{\mathcal{A}_{i}}\right) \gamma\left(N_{s}, \frac{N_{s} x}{2 y}\right) d y, \\
& \mathcal{I}_{2, i}=\frac{1}{\Gamma\left(N_{s}\right)} \int_{\mathcal{A}_{5}}^{\infty} y \exp \left(-\frac{y}{\mathcal{A}_{i}}\right) \gamma\left(N_{s}, \frac{N_{s} x}{2 y}\right) d y .
\end{aligned}
$$

Eqs. (37) and (38), after some basic algebraic manipulations and using [16, Eq. (6.453)], can be written as

$$
\begin{aligned}
\mathcal{I}_{1, i} & =\mathcal{A}_{i} \exp \left(-\frac{\mathcal{A}_{5}}{\mathcal{A}_{i}}\right) \\
& -\frac{\left(N_{s} x\right)^{N_{s} / 2}}{\Gamma\left(N_{s}\right)\left(2 \mathcal{A}_{i}\right)^{\frac{N_{s}}{2}-1}} K_{N_{s}}\left(\sqrt{\frac{2 N_{s} x}{2 \mathcal{A}_{\rangle}}}\right) \\
& +\frac{1}{\Gamma\left(N_{s}\right)} \int_{0}^{\mathcal{A}_{5}} \exp \left(-\frac{y}{\mathcal{A}_{i}}\right) \Gamma\left(N_{s}, \frac{N_{s} x}{2 y}\right) d y
\end{aligned}
$$

and

$$
\begin{aligned}
\mathcal{I}_{2, i} & =\mathcal{A}_{i}^{2} \Gamma\left(2, \frac{\mathcal{A}_{5}}{\mathcal{A}_{i}}\right) \\
& -\frac{1}{\Gamma\left(N_{s}\right)} \int_{0}^{\infty} y^{-3} \exp \left(-\frac{1}{\mathcal{A}_{i} y}\right) \Gamma\left(N_{s}, \frac{N_{s} x y}{2}\right) d y \\
& +\frac{1}{\Gamma\left(N_{s}\right)} \int_{0}^{\mathcal{A}_{5}} y \exp \left(-\frac{y}{\mathcal{A}_{i}}\right) \Gamma\left(N_{s}, \frac{N_{s} x}{y}\right) d y .
\end{aligned}
$$

Using [22, Eq.8.4.16.2] and [22, Eg.2.24.2.1], (40) can be written as

$$
\begin{aligned}
\mathcal{I}_{2, i} & =\mathcal{A}_{i}^{2} \Gamma\left(2, \frac{\mathcal{A}_{5}}{\mathcal{A}_{i}}\right)-\frac{\mathcal{A}_{i}^{2}}{\Gamma\left(N_{s}\right)} G_{1,3}^{3,0}\left[\frac{N_{s} x}{2 \mathcal{A}_{i}} \mid \begin{array}{c}
1 \\
0, N_{s}, 2
\end{array}\right] \\
& +\frac{1}{\Gamma\left(N_{s}\right)} \int_{0}^{\mathcal{A}_{5}} y \exp \left(-\frac{y}{\mathcal{A}_{i}}\right) \Gamma\left(N_{s}, \frac{N_{s} x}{2 y}\right) d y .
\end{aligned}
$$




$$
\begin{aligned}
F_{T_{k}}(x \mid \Theta) & =\sum_{i=2}^{3} U\left(m_{i}-2\right) w_{1, i} w_{2, i}\left(\mathcal{A}_{i} \exp \left(-\frac{\mathcal{A}_{5}}{\mathcal{A}_{i}}\right)-\frac{\left(N_{s} x\right)^{N_{s} / 2}}{\Gamma\left(N_{s}\right)\left(2 \mathcal{A}_{i}\right)^{\frac{N_{s}}{2}-1}} K_{N_{s}}\left(\sqrt{\frac{2 N_{s} x}{2 \mathcal{A}_{i}}}\right)\right) \\
& +\sum_{i=1}^{4} U\left(m_{i}-1\right)\left(U\left(1-m_{i}\right)-\mathcal{A}_{5} U\left(m_{i}-2\right)\right)\left(\mathcal{A}_{i} \exp \left(-\frac{\mathcal{A}_{5}}{\mathcal{A}_{i}}\right)-\frac{\left(N_{s} x\right)^{N_{s} / 2}}{\Gamma\left(N_{s}\right)\left(2 \mathcal{A}_{i}\right)^{\frac{N_{s}}{2}-1}} K_{N_{s}}\left(\sqrt{\frac{2 N_{s} x}{2 \mathcal{A}_{i}}}\right)\right) \\
& +\sum_{i=1}^{4} U\left(m_{i}-1\right) U\left(m_{i}-2\right) w_{1, i}\left(\mathcal{A}_{i}^{2} \Gamma\left(2, \frac{\mathcal{A}_{5}}{\mathcal{A}_{i}}\right)-\frac{\mathcal{A}_{i}}{\Gamma\left(N_{s}\right)} G_{1,3}^{3,0}\left[\frac{N_{s} x}{2 \mathcal{A}_{i}} \mid \begin{array}{c}
1 \\
0, N_{s}, 2
\end{array}\right]\right) \\
& +\sum_{i=2}^{3} \sum_{j=0}^{N_{s}-1} \frac{\left(N_{s} x\right)^{j} U\left(m_{i}-2\right) w_{1, i} w_{2, i}}{2^{j} j !} \int_{0}^{\mathcal{A}_{5}} y^{-j} \exp \left(-\frac{y}{\mathcal{A}_{i}}\right) \exp \left(-\frac{N_{s} x}{2 y}\right) d y \\
& +\sum_{i=1}^{4} \sum_{j=0}^{N_{s}-1} \frac{\left(N_{s} x\right)^{j} U\left(m_{i}-1\right)\left(U\left(1-m_{i}\right)-\mathcal{A}_{5} U\left(m_{i}-2\right)\right)}{2^{j} j !} \int_{0}^{\mathcal{A}_{5}} y^{-j} \exp \left(-\frac{y}{\mathcal{A}_{i}}\right) \exp \left(-\frac{N_{s} x}{2 y}\right) d y \\
& +\sum_{i=1}^{4} \sum_{j=0}^{N_{s}-1} \frac{\left(N_{s} x\right)^{j} U\left(m_{i}-1\right) U\left(m_{i}-2\right) w_{1, i}}{2^{j} j !} \int_{0}^{\mathcal{A}_{5}} y^{-j+1} \exp \left(-\frac{y}{\mathcal{A}_{i}}\right) \exp \left(-\frac{N_{s} x}{2 y}\right) d y .
\end{aligned}
$$

Hence, taking into consideration (37) and (41) and using [16, Eq. (8.352/1)], Eq. (36) results to (34). This concludes the proof.

It should be noted that the calculation of integrals, composed of elementary functions and with finite limits, is involved in the evaluation of (34). This operation can be efficiently performed with numerical integration methods.

Based on the above analysis, the detection probability of the energy detector with RF impairments is

$$
\mathcal{P}_{D}=\sum_{i=1}^{\operatorname{card}\left(\tilde{\Theta}_{1}\right)} P_{r}\left(\tilde{\Theta}_{1}\right)\left(1-F_{T_{k}}\left(\gamma_{t h} \mid \tilde{\Theta}_{1}\right)\right),
$$

where $\tilde{\Theta}_{1}=\left[\theta_{k}=1, \theta_{k-1}, \theta_{k+1}, \theta_{-k+1}, \theta_{-k-1}, \theta_{-k}\right]$. Similarly, the probability of false alarm is

$$
\begin{array}{r}
\mathcal{P}_{F A}=\sum_{i=1}^{\operatorname{card}\left(\tilde{\Theta}_{2, c}\right)} \operatorname{Pr}\left(\tilde{\Theta}_{2}\right)\left(1-F_{T_{k}}\left(\gamma_{t h} \mid \tilde{\Theta}_{2, c}\right)\right) \\
+\operatorname{Pr}\left(\tilde{\Theta}_{2,0}\right)\left(1-\frac{\gamma\left(N_{s}, \frac{N_{s} \gamma_{t h}}{2 \mathcal{A}_{5}}\right)}{\Gamma\left(N_{s}\right)}\right),
\end{array}
$$

where $\tilde{\Theta}_{2,0}=[0,0,0,0,0,0], \tilde{\Theta}_{2, c}=\tilde{\Theta}_{2}-\tilde{\Theta}_{2,0}$, and $\tilde{\Theta}_{2}=$ $\left[\theta_{k}=0, \theta_{k-1}, \theta_{k+1}, \theta_{-k+1}, \theta_{-k-1}, \theta_{-k}\right]$.

\section{NUMERICAL RESULTS}

In this section, we show the effects of RF impairments on the spectrum sensing performance of EDs by illustrating analytical and Monte-Carlo simulation results for different RF imperfection levels. In particular, we consider the following insightful scenario. It is assumed that there are $K=8$ channels and the second channel is sensed. The signal and the total guard band bandwidths are assumed to be equal with $W_{s b}=1$ $\mathrm{MHz}$ and $W_{g b}=125 \mathrm{KHz}$, respectively, while the sampling rate is chosen to be equal to the bandwidth of wireless signal as $W=9 \mathrm{MHz}$. Morevoer, the channel occupancy process is assumed to be Bernoulli distributed with probability, $q=1 / 2$, and independent across channels, while the signal variance is equal for all channels. Finally, the number of samples used for detection is set to $5\left(N_{s}=5\right)$ and the variance of the fading process is assumed to be equal with unity $\left(\sigma_{h}^{2}=1\right)$. It should be noted that each of the following figures contains both analytical and simulation results, which are represented by lines and discrete marks, respectively.

Fig. 1 demonstrates the impact of IBO non-linearities on the performance of the classical energy detector, assuming various SNR values. Specifically, receiver operation curves (ROC) are plotted for different SNR and IBO values, considering $100 \mathrm{~Hz} 3-\mathrm{dB}$ bandwidth and IRR and phase imbalance equal to $25 \mathrm{~dB}$ and $\phi=3^{\circ}$, respectively. As a benchmark, the ROC curve of an ED with ideal RF FE is also plotted. It becomes evident from the figure that the analytical results are identical with simulation results; thus, verifying the the presented analytical framework. Furthermore, it is observed that at low SNR values, IBO non-linearitues do not affect the spectrum sensing capability of the ED compared to the ED with ideal RF FE. However, as SNR increases, IBO nonlinearities become to have more detrimental effects on the spectrum capabilities of the ED, significantly reducing its performance for low IBO values.

The effects of IQI on the spectrum sensing performance of $\mathrm{ED}$ are illustrated at Fig. 2. In particular, this figure illustrates ROC curves assuming various SNRs, when the $3-\mathrm{dB}$ bandwidth and IBO are set to $100 \mathrm{~Hz}$ and $3 \mathrm{~dB}$, respectively. Again, it becomes evident from the figure that analytical results coincide with simulation results. Moreover, at low SNRs, it is observed that there is no significant performance degradation due to I/Q imbalance. However, as SNR increases, this RF imperfection notably affects the spectrum sensing performance. When compared with the degradation caused by LNA nonlinearities, it becomes apparent that the impact of LNA nonlinearity to the spectrum sensing performance becomes more detrimental than the impact of IQI. 


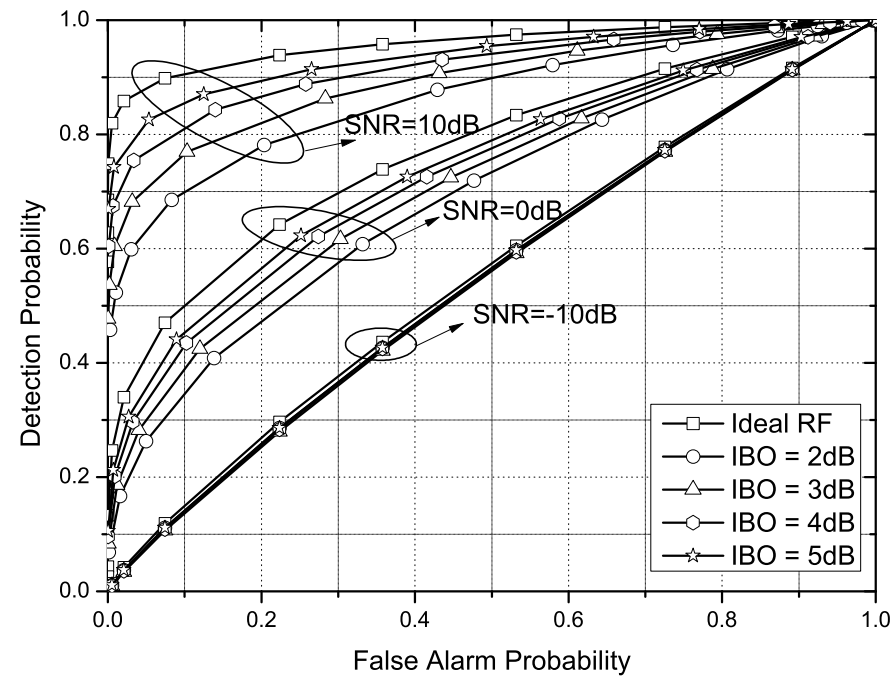

Fig. 1: ROC curves for different IBO and SNR values, assuming $\beta=100 \mathrm{~Hz}$ and $I R R=25 \mathrm{~dB}$.

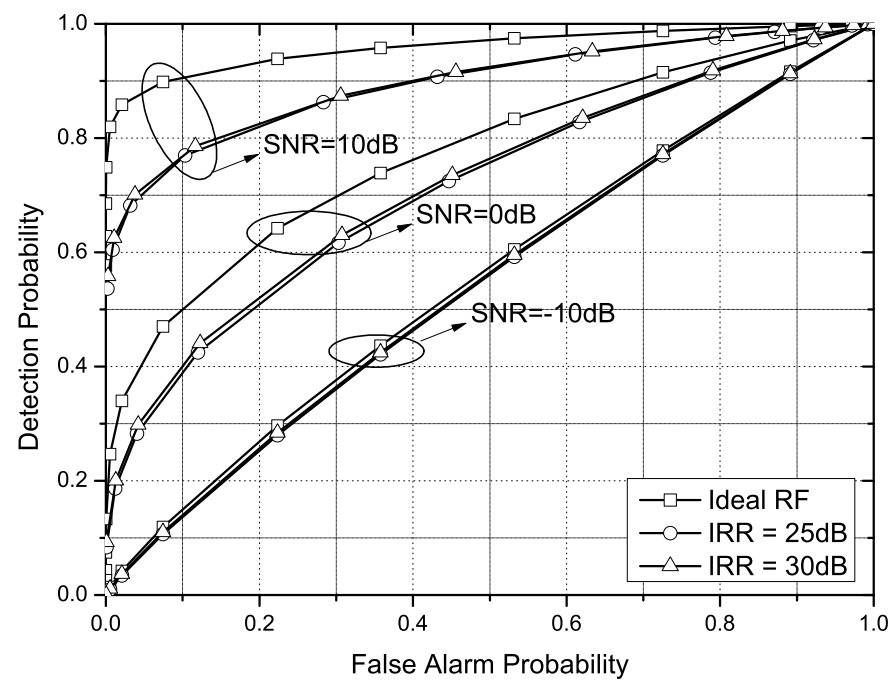

Fig. 2: ROC curves for different IRR and SNR values, assuming $\beta=100 \mathrm{~Hz}$ and $I B O=3 \mathrm{~dB}$.

\section{CONCLUSIONS}

We studied the performance of multi-channel spectrum sensing, when the RF FE is impaired by hardware imperfections. In particular, assuming Rayleigh fading, we provided the analytical framework for evaluating the detection and false alarm probabilities of energy detectors when LNA nonlinearities, IQI and PHN are taken into account. Our results illustrated the degrading effects of RF imperfections on the ED spectrum sensing performance, which bring significant losses in the utilization of the spectrum. Therefore, they should be seriously taken into consideration when designing direct conversion CR receivers. Moreover, among others, LNA non-linearities were shown to have the most detrimental effect on the spectrum sensing performance. In our ongoing research, we shall investigate energy detectors that take into consideration the parameters of RF imperfections and enhance their spectrum sensing capabilities.

\section{REFERENCES}

[1] FCC, "Spectrum policy task force report," November 2002

[2] T. Yucek and H. Arslan, "A survey of spectrum sensing algorithms for cognitive radio applications," IEEE Communications Surveys \& Tutorials, vol. 11, no. 1, pp. 116-130, March 2009.

[3] O. Altrad, S. Muhaida, A. Al-Dweik, A. Shami, and P. D. Yoo, "Opportunistic spectrum access in cognitive radio networks under imperfect spectrum sensing," IEEE Trans. Veh. Technol., vol. 63, no. 2, pp. 920925, Feb 2014

[4] L. Fan, X. Lei, T. Q. Duong, R. Q. Hu, and M. Elkashlan, "Multiuser cognitive relay networks: Joint impact of direct and relay communications," IEEE Trans. Wireless Commun., vol. 13, no. 9, pp. 5043-5055, Sept 2014.

[5] S. Huang, X. Liu, and Z. Ding, "Optimal sensing-transmission structure for dynamic spectrum access," in IEEE INFOCOM, April 2009, pp. 2295-2303.

[6] S. Atapattu, C. Tellambura, and H. Jiang, "Energy detection based cooperative spectrum sensing in cognitive radio networks," IEEE Trans. Commun., vol. 10, no. 4, pp. 1232-1241, April 2011.

[7] M. Z. Shakir, A. Rao, and M.-S. Alouini, "Generalized mean detector for collaborative spectrum sensing," IEEE Trans. Commun., vol. 61, no. 4, pp. 1242-1253, April 2013.

[8] A. Gokceoglu, S. Dikmese, M. Valkama, and M. Renfors, "Energy Detection under IQ Imbalance with Single- and Multi-Channel DirectConversion Receiver: Analysis and Mitigation," IEEE J. Sel. Areas Commun., vol. 32, no. 3, pp. 411-424, March 2014.

[9] B. Razavi, "Cognitive radio design challenges and techniques," IEEE $J$. Solid-State Circuits, vol. 45, no. 8, pp. 1542-1553, Aug 2010.

[10] A. Gokceoglu, Y. Zou, M. Valkama, and P. C. Sofotasios, "Multi-channel energy detection under phase noise: analysis and mitigation," Mobile Networks and Applications, May 2014.

[11] J. Verlant-Chenet, J. Renard, J.-M. Dricot, P. D. Doncker, and F. Horlin, "Sensitivity of spectrum sensing techniques to RF impairments," in IEEE 71st Vehicular Technology Conference (VTC 2010-Spring), May 2010, pp. 1-5.

[12] A. Zahedi-Ghasabeh, A. Tarighat, and B. Daneshrad, "Cyclo-stationary sensing of OFDM waveforms in the presence of receiver RF impairments," in IEEE Wireless Communications and Networking Conference (WCNC), April 2010, pp. 1-6.

[13] A. ElSamadouny, A. Gomaa, and N. Al-Dhahir, "Likelihood-based spectrum sensing of OFDM signals in the presence of Tx/Rx I/Q imbalance," in IEEE Global Communications Conference (GLOBECOM), Dec 2012, pp. 3616-3621.

[14] O. Semiari, B. Maham, and C. Yuen, "Effect of $I / Q$ imbalance on blind spectrum sensing for ofdma overlay cognitive radio," in 1st IEEE International Conference on Communications in China (ICCC), Aug 2012, pp. 433-437.

[15] J. Li, M. Matthaiou, and T. Svensson, "I/Q imbalance in af dual-hop relaying: Performance analysis in Nakagami-m fading," IEEE Trans. Commun., vol. PP, no. 99, pp. 1-12, 2014.

[16] I. S. Gradshteyn and I. M. Ryzhik, Table of Integrals, Series, and Products, 6th ed. New York: Academic, 2000.

[17] T. Schenk, RF Imperfections in High-Rate Wireless Systems. The Netherlands: Springer, 2008.

[18] A. Papoulis and S. Pillai, Probability, Random Variables, and Stochastic Processes, ser. McGraw-Hill series in electrical engineering: Communications and signal processing. Tata McGraw-Hill, 2002.

[19] S. Mirabbasi and K. Martin, "Classical and modern receiver architectures," IEEE Commun. Mag., vol. 38, no. 11, pp. 132-139, Nov 2000.

[20] Y. Zhou and Z. Pan, "Impact of LPF Mismatch on I/Q Imbalance in Direct Conversion Receivers," IEEE Trans. Wireless Commun., vol. 10, no. 6, pp. 1702-1708, June 2011.

[21] G. K. Karagiannidis, N. C. Sagias, and T. A. Tsiftsis, "Closed-form statistics for the sum of squared Nakagami-m variates and its applications," IEEE Trans. Commun., vol. 54, pp. 1353-1359, 2006.

[22] A. Prudnikov, Y. Brychkov, and O. Marichev, Integrals and series. Gordon and Breach Science Publishers, 1986, vol. 2. 